\title{
Pathophysiological and Psychosocial Features in Functional Vomiting: Author's Reply
}

TO THE EDITOR: We appreciate the comments from Kang. This study was conducted to further understand the pathophysiological and psychosocial features in functional vomiting (FV). Patients diagnosed as FV should fulfill the Rome III criteria, ${ }^{1}$ which does not require the absence of other functional gastrointestinal disorders. Gastrointestinal symptoms in all FV patients were recorded by questionnaires, which included vomiting symptoms, other upper and lower gastrointestinal symptoms. Recent studies have revealed that overlaps are common in patients with functional gastrointestinal disorders and about two thirds have symptoms of multiple disorders at the same time. ${ }^{2,3}$ I agree the pathophysiology in our study might reflect both the FV and functional dyspepsia. However, our FV patients presented vomiting as a predominant symptom.

Electrogastrography was used to investigate gastric electric activity instead of gastric emptying study since we wanted to avoid vomiting during testing. Power ratio is the ratio of the postprandial dominant power (DP) divided by the fasting DP. Although average DP increased, there was still $50 \%$ abnormal power ratio $(<1)$, suggesting a poor motor response to the meal.

In our study, psychosocial factors played an important role in FV patients in our study and we paid more attention to the relationship between psychosocial factors and the amount of overlaps or gastric sensorimotor function.

Electric barostat has been regarded as gold standard method to evaluate the gastric accommodation and visceral sensitivity. ${ }^{4}$ However, it is not physiological, and not available in clinic. Instead, drinking test is easier to perform. ${ }^{5}$ Most recently, our perfusion nutrition load test (P-NLT) was shown to reflect gastric accommodation more accurately than the drinking test in health subjects and functional dyspepsia. ${ }^{6}$ Therefore, we used P-NLT in our present study. Further studies are needed to confirm the validation of this method. In addition, further studies with large cases and other sensormotor function test are needed to reinforce the findings in our study.

Yiming Zhao, MD Meiyun Ke, MD

Department of Gastroenterology Peking Union Medical College Hospital Chinese Academy of Medical Sciences Beijing, China

1. Tack J, Talley NJ, Camilleri M, et al. Functional gastroduodenal disorders. Gastroenterology 2006;130:1466-1479.

2. Corazziari E. Definition and epidemiology of functional gastrointestinal disorders. Best Pract Res Clin Gastroenterol 2004;18:613631.

3. Drossman DA, Li Z, Andruzzi E, et al. U.S. householder survey of functional gastrointestinal disorders. Prevalence, sociodemography, and health impact. Dig Dis Sci 1993;38:1569-1580.

4. Zhang J, Sun SH, Ke MY. Evaluation of measurements for proximal gastric accommodation and its clinical application. Chin J Gastroenterol 2010;15:492-495.

5. Shao C, Zhao H, Ke MY, et al. Role of water load test combined with ultrasound in the proximal stomach of functional dyspepsia. Chin J Digestion 2005;25:23-26.

6. Zhang J, Sun XH, Wang ZF, et al. Perfusion nutrient load test - a new method in assessment of gastric accommodation. Chin J Digestion 2010;30:518-521.

\section{Conflicts of interest: None.}

